\title{
Gas and Dust in Ultraluminous Infrared Galaxies: Implications for Sources at High Redshift
}

\section{Downes}

\section{Institut de Radio Astronomie Millimétrique, Grenoble, France}

\begin{abstract}
High- $z \mathrm{~mm}$ and submm dust sources are thought to be similar to ultraluminous infrared galaxies, where merger interactions have driven most of the gas and dust into circumnuclear molecular disks or rings with typical radii of $500 \mathrm{pc}$. Within these disks, $\mathrm{CO}$ and radio data indicate a new class of extreme starbursts, with characteristic sizes of $100 \mathrm{pc}$, gas masses of $10^{9} \mathrm{M}_{\odot}$ and IR luminosities of $\sim 3 \times 10^{11} L_{\odot}$ from OB stars. Some of these regions, however, harbor powerful AGNs, even though the optical IR diagnostics may classify them as starbursts. Trentham (2000) argues that averaged over time, most of the high- $z$ dust sources must be powered by AGNs.
\end{abstract}

The high-redshift $\mathrm{mm}$ and submm dust sources have IR luminosities and SEDs similar to the local-universe ultraluminous infrared galaxies (ULIGs or ULIRGs; for a recent overview, see the proceedings of the 1998 Ringberg ULIG conference, edited by Lutz \& Tacconi 1999). I review here some new ULIG results that have appeared since that conference, and the implications for interpreting the $\mathrm{mm}$ and submm detections at high redshift.

Major new data papers have appeared recently. Soifer et al. (2000) present 8 to $25 \mu \mathrm{m}$ images of 7 ULIGS from the Keck Telescopes with $0 " 3$ to $0 " 6$ resolution. The $25 \mu \mathrm{m}$ maps give the closest approximation to what the sources would look like at $100 \mu \mathrm{m}$ if we could get 0.5 resolution at $100 \mu \mathrm{m}$. For example, in Arp 220 (Soifer et al. 1999) one interpretation of the $25 \mu \mathrm{m}$ map is that $\sim 75 \%$ of the total luminosity of the galaxy comes from the western peak.

Scoville et al. (2000) give HST NICMOS-camera images of 24 luminous IR galaxies, including 15 ULIGs, with resolution $<0^{\prime \prime} .2$. These are the highestresolution ULIG images available in the near-IR. The data are given as separate maps at $1.1,1.6$, and $2.2 \mu \mathrm{m}$, as "true color" combined images of the three bands, and as ratio (color) maps, that show the regions of strongest extinction in the near-IR. There are some surprises, like the remarkable mini-spiral in the nucleus of VII Zw 31, which on ground-based images resembles a large elliptical galaxy.

Surace \& Sanders (2000) present near-UV $(3410 \AA)$ images of 20 ULIGS. These images are important because they give an idea what ULIGs at $z=1$ would look like at $6800 \AA$, or what ULIGs at $z=2$ to 4 ought to look like in deep field images in the HST NICMOS bands.

Extreme starbursts: There is new evidence for extreme starbursts as substructure within the main ULIG dust and gas concentration. These are compact, 80 to 100 -pc regions with strong, but extended, non-thermal radio continuum and compact radio supernovae, such as those found in the east and 
west "nuclei" of Arp 220 (Smith et al. 1998). Another example is the group of radio supernovae in Mrk 273, seen at $1.4 \mathrm{GHz}$ with the VLBA (Carilli \& Taylor 2000) - all within a bright compact CO source suggestive of an extreme starburst region (Downes \& Solomon 1998). The innermost parts of the rotating $\mathrm{CO}$ disk, are shown in MERLIN maps of $\mathrm{HI}$ absorption and $\mathrm{OH}$ megamasers (Cole et al. 1999; Yates et al. 2000).

Transition objects: There are new results on IR "warm" ULIGs, in which the black hole accretion luminosity, re-radiated by a dust torus in the NIR, is a large part of the power output. Some of these objects are classed both as ULIGs and as quasars. The prototype is Mrk 231, for which Keck observations (Soifer et al. 2000) yield a $12.5 \mu \mathrm{m}$ size upper limit of $<0 " 1(3 \sigma, 100 \mathrm{pc})$, and a $60 \mu \mathrm{m}$ size much larger than the $12.5 \mu \mathrm{m}$ size. Soifer et al. note that the increase in size of the IR source with wavelength is consistent with an AGN of luminosity $3 \times 10^{12} L_{\odot}$ heating the surrounding dust and producing a radial temperature gradient. Against this interpretation however, are $a$ ) the low power of the central AGN in hard X rays $\left(8 \times 10^{8} L_{\odot}\right.$, or $\leq 1 \%$ of the bolometric luminosity; Nakagawa et al. 1999); $b$ ) the properties of the 350-pc radio continuum disk (short electron lifetimes, flat spectrum, consistency with the radio-FIR relation for starbursts; Ulvestad, Wrobel, \& Carilli 1999); c) the free-free absorption of the disk at low frequencies, the 4 candidate radio supernovae in the disk (Taylor et al. 1999); $d$ ) the sum of X-ray, UV, visible, and NIR luminosities, all corrected for extinction, that yields $\leq 30 \%$ of the bolometric luminosity (Downes \& Solomon 1998).

New HST images of the IR "warm" ULIGs Mrk 231, Mrk 1014, and IRAS 07598+6508 (Canalizo \& Stockton 2000) show the central quasars are surrounded by near-nuclear starbursts, for which photometry compared with starburst templates yield relatively young ages. In Mrk 231, Canalizo \& Stockton derive an age of $\leq 40 \mathrm{Myr}$ for the starburst $3 \mathrm{kpc}$ south of the nucleus. The starburst regions in the central $1 \mathrm{kpc}$ around the quasar are very similar in both size and brightness, and may also be as young as the region at $r=3 \mathrm{kpc}$. The near-in starbursts are within the molecular disk mapped in CO (Bryant \& Scoville 1996; Downes \& Solomon 1998).

NGC 6240: While Arp 220 is often called the ULIG prototype, the luminous $\left(7 \times 10^{11} L_{\odot}\right)$ merging galaxy NGC 6240 is even more emblematic of the starburst-AGN debate. This merger shows dramatic tidal tails over a region $>100 \mathrm{kpc}$. Although the mid-IR lines observed by ISO would class this object as starburst-powered (Genzel et al. 1998; Rigopoulou et al. 1999), it contains a powerful AGN, as shown by its high luminosity in hard $\mathrm{X}$ rays $\left(\sim 10^{11} L_{\odot}\right)$ and its $6.4 \mathrm{keV}$ iron K-shell lines (e.g., Komossa \& Schulz 1999; Nakagawa et al. 1999; Vignati et al. 1999; Ikebe et al. 2000). The HST NICMOS color map (Scoville et al. 2000) indicates maximum extinction between the two IR nuclei of NGC 6240. Tecza et al. (2000) mapped the $2 \mu \mathrm{m}$ CO band head emission across the two $\mathrm{K}$-band peaks separated by $1 " 6$ to derive the rotation velocity and the velocity dispersion of the stars in the two nuclei. Both of these velocity measures correspond to large masses, much greater than that implied by the $\mathrm{K}$-band light, which is dominated by red supergiants. These large masses imply that the K-band peaks are the massive, pre-merger nuclei, with the velocities indicating dynamical masses of (2 to 8$) \times 10^{9} M_{\odot}$, mostly in old stars, within the central $500 \mathrm{pc}$ of each nucleus. From their models of the K-band luminosity, 
Tecza et al. derive a mass of $(0.4$ to 2$) \times 10^{8} M_{\odot}$, of new stars, formed in nuclear starbursts that lasted $5 \mathrm{Myr}$, about 15 to $25 \mathrm{Myr}$ ago. The gas, however, as traced in the $\mathrm{mm}$ lines of $\mathrm{CO}$, is between the two K-band peaks, as if it has been driven by the two nuclei into the gravitational potential minimum between them (Tacconi et al. 1999; Bryant \& Scoville 1999). The interesting question is: where is the X-ray emitting supermassive black hole? - in the former nuclear bulges, or in the self-gravitating gas cloud in the middle? And does it power most of the far-IR luminosity of NGC 6240 ?

Trentham's Argument: An interesting argument has been formulated by Trentham (2000). From their bolometric luminosity and spectral energy distribution, the submm dust sources detected with SCUBA appear to be the high- $z$ analogs of the local-universe ULIGs. The only realistic end product of the ULIGs are cores of giant elliptical $(\mathrm{gE})$ galaxies, and their supermassive black holes. The current ULIG number density is $1.1 \times 10^{-7} \mathrm{Mpc}^{-3}$, but that of giant elliptical galaxies is $1.6 \times 10^{-4}$, most of which have cores and supermassive black holes, which means that their progenitors, the high- $z$ ULIGs, were 1500 times as numerous at earlier epochs $(z>2)$, consistent with the universe being smaller and mergers more frequent. The number density of high- $z$ SCUBA sources is $10^{-4} \mathrm{Mpc}^{-3}$, so the story seems to fit. This explains the local number density of $\mathrm{gE}$ cores and supermassive black holes $\left(10^{-4} \mathrm{Mpc}^{-3}\right)-$ they were the SCUBA sources at earlier epochs. This picture not only explains the local number density of $\mathrm{gE}$ cores, but also their size (radius several hundred $\mathrm{pc}$ ), stellar density $\left(\sim 100 M_{\odot} \mathrm{pc}^{-3}\right)$, and the fact that they are often kinematically decoupled from the rest of the giant elliptical. Furthermore, $M_{\mathrm{BH}} \propto \sigma^{4}$, where $M_{\mathrm{BH}}$ is the mass of the supermassive black hole, and $\sigma$ is the velocity dispersion of the central spheroid, implying that the mass of the black hole is one-third the mass of the $\mathrm{gE}$ core.

Trentham's argument is that if the high-z SCUBA sources have the same gas and dust distributions as local ULIGs, then, averaged over time, they must be powered by AGNs. The reason is that the ratio of accretion to nucleosynthesis luminosities per unit mass is $1760 \eta$, where $\eta$ is the efficiency of accretion luminosity, often taken to be 0.1 . Since one-third of the core mass accretes onto the black hole, the net result is that, averaged over time, the accretion luminosity should be larger than the star formation luminosity by the factor $560 \eta$. Trentham supports his conclusion that the SCUBA sources must be powered by AGNs by noting that they give a bolometric background radiation of $7 \mathrm{nW} \mathrm{m}^{-2} \mathrm{sr}^{-1}$, or $10 \%$ of the total bolometric luminosity of the universe, and about that expected from the production of supermassive black holes in $\mathrm{gE}$ cores, and also about that expected if the hard X-ray background comes from absorbed quasars that re-radiate at far-IR and submm wavelengths (Fabian \& Iwasawa 1999; Almaini et al. 1999). This is in strong contrast to what the SCUBA sources could give by star formation, if the SCUBA sources have the same concentrated gas distribution as the local ULIGs. Since then their only realistic end products would be the $\mathrm{gE}$ cores, the SCUBA sources would form $<1 \%$ by mass of the stars in the universe. Hence their large power output would have to come from AGNs, not stars. Hence the SCUBA sources would not be the same as the local-universe ULIGs, for which only $1 / 3$ appear to be powered by AGNs. 
So far, however, the SCUBA observations of the CHANDRA deep X-ray field show that the high- $z$ dust sources are generally not the same objects as the AGN X-ray sources (Barger et al. 2000), which may mean that they are not powered by AGNs, in contradiction to Trentham's argument. Are the AGNs heavily obscured, as in NGC 6240 ?

\section{References}

Almaini, O., Lawrence, A. \& Boyle, B.J. 1999, MNRAS, 305, L59

Barger, A.J., Cowie, L.L., Mushotzky, R.F. \& Richards, E.A. 2000, AJ, in press (astro-ph/0007175)

Bryant, P.M. \& Scoville, N.Z. 1999, AJ, 117, 2632

Canalizo, G. \& Stockton, A. 2000, AJ, 120, 1750

Carilli, C.L. \& Taylor, G.B. 2000, ApJ, 532, L95

Cole, G.H.J., Pedlar, A., Holloway, A.J. \& Mundell, C.G. 1999, MNRAS, 316, 1033

Downes, D. \& Solomon, P.M. 1998, ApJ, 507, 615

Fabian, A. \& Iwasawa 1999, MNRAS, 303, L34

Genzel, R., et al. 1998, ApJ, 498, 579

Ikebe, Y., Leighly, K., Tanaka, Y., Nakagawa, T., Terashima, Y. \& Komossa, S. 2000, MNRAS, 316, 433

Komossa, S. \& Schulz, H. 1999, Ap\&SS, 266, 61

Lutz, D. \& Tacconi, L.J., (eds.) Ap\&SS, 266/1-2

Nakagawa, T., Kii, T., Fujimoto, R., Miyazaki, T., Inoue, H., Ogasaka, Y. \& Kawabe, R. 1999, Ap\&SS, 266, 43

Rigopoulou, D., Spoon, H.W.W., Genzel, R., Lutz, D., Moorwood, A.F.M. \& Tran, Q.D. 1999, AJ, 118, 2625

Scoville, N.Z., et al. 2000, AJ, 119, 991

Smith, H.E., Lonsdale, C.J., Lonsdale, C.J. \& Diamond, P.J. 1998, ApJ, 493, L17

Soifer, B.T., et al. 1999, ApJ, 513, 207

Soifer, B.T., et al. 2000, AJ, 119, 509

Surace, J.A. \& Sanders, D.B., 2000, AJ, 120, 604

Tacconi, L.J., Genzel, R., Tecza, M., Gallimore, J.F., Downes, D. \& Scoville, N.Z. 1999, ApJ, 524, 732

Taylor, G.B., Silver, C.S., Ulvestad, J.S. \& Carilli, C.L. 1999, ApJ, 519, 185

Tecza, M., Genzel, R., Tacconi, L.J., Anders, S., Tacconi-Garman, L.E. \& Thatte, N. 2000, ApJ, 537, 178

Trentham, N. 2000, MNRAS, in press (astro-ph/0004370)

Ulvestad, J.S., Wrobel, J.M. \& Carilli, C.L. 1999, ApJ, 516, 127

Vignati, P., et al. 1999, A\&A, 349, L57

Yates, J.A., Richards, A.M.S., Wright, M.M., Cohen, J.L., Gray, M.D., Field, D. \& Cohen, R.J. 2000, MNRAS, 317, 28 